\title{
Work-related risk associated with musculoskeletal disorders amongst flower farm workers in Kenya
}

\section{J. M. Munala*1, B. Olivier ${ }^{2}$.}

${ }^{*}$ Department of Physiotherapy, Faculty of Rehabilitative Sciences, Kenya Medical Training College, Nairobi, Kenya.

${ }^{2}$ Department of Physiotherapy, Faculty of Health Sciences, University of the Witwatersrand, Pretoria, South Africa.

\section{ABSTRACT}

Background: Work-related musculoskeletal disorders (WRMSDs) are a public concern to social-care and health systems, as well as individuals. Globally, WRMSDs are classified as one of the prevalent causes of disability.

Objectives: The primary objective of this study was to determine work-related risk associated with musculoskeletal disorders amongst flower farm workers. The secondary objective was to determine the relationship between WRMSD risk over the previous 12 months and socio-demographic characteristics.

Method: A cross-sectional descriptive study was conducted. A sample of 270 participants was drawn from 897 farm workers, of which 184 presented with WRMSD as assessed using the Nordic Musculoskeletal Questionnaire. Quantitative data were collected using the Rapid Entire Body Assessment questionnaire. Inferential statistics were analyzed using the Pearson's chi-squared test $\left(X^{2}\right)$ test and based on an alpha level of $p<0.05$. Descriptive statistics were presented using frequencies and percentages.

Results: Respondents who reported medium risk were 49 (26.6\%), 80 (43.5\%) reported high risk while 55 (29.9\%) reported very high risk. There was no relationship between WRMSD risk and socio-demographic characteristics.

Conclusions: Flower farm workers in Kenya were found to be at risk of sustaining WRMSDs although socio-demographic factors did not play a role in the level of risk.

Clinical Implications: The high prevalence of WRMSDs necessitates policy reform in the flower farm industry. Furthermore, the timely identification of potential disorders and the associated risk factors is necessary to ensure early intervention.

Key words: Work-related risk, Work-related musculoskeletal disorders, Farm workers.

Address for correspondence: J. M. Munala, Department of Physiotherapy, Faculty of Rehabilitative Sciences, Kenya Medical Training College, Nairobi, Kenya.

E-Mail: jothampt@gmail.com

Access this Article online

Quick Response code

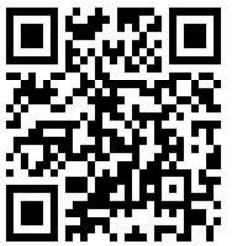

DOI: $10.16965 /$ ijpr.2021.120

Journal Information

International Journal of Physiotherapy and Research ISSN (E) 2321-1822 | ISSN (P) 2321-8975

https://www.ijmhr.org/ijpr.html DOI-Prefix: https://dx.doi.org/10.16965/ijpr

(c) $\mathrm{Er}+\mathrm{Mr}-\mathrm{BA}$

Article Information

Received: 03 Mar 2021

Peer Review: 04 Mar 2021

Revised: None
Accepted: 04 Apr 2021

Published (O): 11 Jun 2021

Published (P): 11 Jun 2021

\section{INTRODUCTION}

Agriculture is not only a backbone of economy in Kenya but also play a large role in the global economy. Due to the need of high volume of farm-production, there is a counter need to have an increased working pace in the farms. This predisposes farm workers to workrelated musculoskeletal disorders (WRMSDs) which are the greatest in prevalence of all of all non-fatal disorders [1]. The level of complexity and variety of the risk factors associated with WRMSD clearly explains the 
J. M. Munala, B. Olivier. Work-related risk associated with musculoskeletal disorders amongst flower farm workers in Kenya.

challenges faced while trying to address the ergonomic prescription, intervention, and management of WRMSD [2].

This WRMSD is said to be an occupationalcaused illness because it occurs over a period of time mostly among a working population [3], and arise due to the body's exposure to pivoting load at work, poor-working posture, repetitive movements, poor working environment and long static standing [4-6]. It is mostly characterized by aching stiffness, numbness or tingling sensation, discomfort, pain, and fatigue [7]. At advanced stages, they present with decreased and or impaired function and mobility [4]. The overall physical nature of agricultural activities poses serious health consequences [8]. As noted earlier, WRMSDs may advance to chronic severe phases, treatment and recovery are often unsatisfactory among most subjects, thus disability and even loss of employment $[7,9]$.

White-collar careers such as the healthcare, teaching, engineering and banking professions also expose individuals to work-related risk of developing WRMSDs, and have varying levels of work-related risk. Workers in agriculture, forestry, construction, human health, transport, and storage are, however, affected to a larger degree [10]. Work-related risk are usually due to factors broadly categorized into three sets, namely: 'Individual factors' such as sports activity level, previous WRMSD, recreational activities, age, professional activities and length of service, education levels, gender, BMI, smoking or alcohol behaviors, marital status, social class, monthly income. The second set is 'Physical factors': hand-arm vibration, mechanical compression, forceful exertions, sustained posture, awkward posture, and repetitive activities. Lastly, 'Psychosocial factors' such as support from team or boss at work, autonomy, lack of job control, job uncertainty, work pace, monotony, work-rest cycles, time pressure, and overall social support [5, 11-16]. A study [11] reported that workers in the age group of $46-55$ years were the most affected. In overall, most patients who report WRMSD have a link between the duration they had worked, age, the specialty of work, and weight [17-18].
An occupational study [19] further revealed that the longer individuals were exposed to an occupation, the more likely they were to suffer WRMSD. A study [20] found a strong association of developing WRMSDs with gender, the period that one has worked and age. The same results were also concurred with [10]. In a study conducted in an apparel firm in Uganda [21], the female gender reported the highest risk. This is was attributed to the plentiful responsibilities they had to attend to besides the duty at the Apparel firm.

An agricultural safety report [10] reported that agricultural activities rank among the highest occupational hazards globally and that, farmers are at very high risk for both fatal and nonfatal injuries. With the current high industrialization levels in agriculture, new farming equipment has been introduced, such as load-transfer devices [22], but limited attention has been paid to the overall ergonomic design and their usage [1]. Besides, prolonged working duration and working pace as risk factors have been brought about by increased work demands and competition to produce in large quantities especially in low- and middle-income countries [23].

Advances in worker-based approaches such as load transfer, if well utilized, give a great promise in curbing the prevalent culture of prolonged stooping while working on the farm. However, even in the advent of these new technologies in farming, reliance on human labor are still expected. Further, the overall prevalence of WRMSDs could be significantly reduced if proper technical advancements were adopted [24]. It is worth noting that advancement in research in agriculture and farming is taking new and better heights among various professionals such as Epidemiologists, Occupation and Health professionals, social scientists among others globally are committed to the sole role of reducing WRMSDs and other health issues among farmers [22].

Despite horticultural farming activities being a major commercial sector in Kenya, there is no published research has been conducted on WRMSDs and work-related risk. Although many tools have been innovated to make work friendly, easier and reduce work-related risk, 
J. M. Munala, B. Olivier. Work-related risk associated with musculoskeletal disorders amongst flower farm workers in Kenya.

there are still many challenges in their utilization such as in picking flowers, weeding planting, and overall crop management [7].

Therefore, the primary objective of this study was to determine work-related risk associated with WRMSDs among flower farm workers. The secondary objective was to determine the relationship between WRMSDs risk over the previous 12 months and socio-demographic characteristics. In understanding the risk levels the farm workers are exposed to, the farm management may be conscious to initiate WRMSD risk scheduled-screening programs and take appropriate measures to reduce the risk levels such as setting an environment that is occupational friendly, introducing load transfer devices, and creating overall awareness on proper ergonomics.

\section{METHODS}

Study design: A descriptive cross-sectional study design was utilized.

Population: The focus of the business at a flower farm is to grow flowers for foreign markets. There are only two job positions in the farm, which are laborious and manual in mechanism. The general workers execute less specialized farm duties such as cutting flowers, pruning, planting, weeding and transporting flowers from the farm. General farm maintenance duties such nurseries and green houses were also their duties. The total number of flower farm workers employed at the farm were 897. The other cadre is that of the Sprayers who carried out spraying tasks. The formula $n=Z^{2} p q / d^{2}$ [25] was used to for determine the sample size. The calculated sample size was 270 (30\%) farm workers. The systematic sampling method was used to select the individual farm workers from a single continuous list of all farm workers generated according to their names arranged in alphabetical order.

Selection criteria: Farm workers who were aged 18 years and above but had worked for at least four months in flower farming were included in the study in order to more accurately determine an association between exposure to farming and developing musculoskeletal disorders. Farm workers who had joined the horticultural farming industry with pre-existing musculoskeletal disorders as well as those who were pregnant were excluded.

Data collection tool: The Nordic Musculoskeletal Questionnaire (NMQ) is cross-culturally adapted questionnaire which was used to screen WRMSDs [26]. The specificity and sensitivity of NMQ ranged from 71 and $88 \%$, and 66 and 92\%, respectively [26]. Sociodemographic characteristics studied and captured by this tool were gender, age, weight, job designation and the length of time the farm worker had been working at the time of the research. All of the 270 respondents completed the questionnaire with responses as to whether they had experienced musculoskeletal pain in any part of the body over the previous 12 months.

The Rapid Entire Body Assessment (REBA) method is an observational ergonomic assessment tool for the analysis of individuals' body during work and evaluates the risk of WRMSDs. It focused on the range of motion, direction of joints motion, the amount of force exerted, the load grip condition, and the activity. It is scored by assigning numbers to body areas for accumulation, analysis, and interpretation [27, 28].

Reliability of the REBA method where interobserver reliability between the 14 participants for coding was found to be between $62 \%$ and $85 \%$. Further work is needed to establish the validity of the tool [29]. Initially, REBA has been used to assess ergonomics in farming [30, $31,32,33]$.

Data analysis and management: All the questionnaires were securely kept by the principal researcher in a lockable metal box. Data from both the NMQ and the REBA and was keyed into the Microsoft Excel spreadsheet database and secured using passwords known only to the principal researcher. SPSS version 25 was used for statistical analysis. Inferential statistics of associations between WRMSD risk levels and the variables were then analyzed using the Pearson Chi-square $\left(\mathrm{X}^{2}\right)$ test and based on an alpha level of $p<0.05$. Descriptive statistics were described by percentages and frequencies. 
Procedure: The researcher acquired approval from: Jomo Kenyatta University of Agriculture and Technology - Institutional Review and Ethics Committee (JKU/2/4/896B), The National Commission for Science Technology and Innovation (Ref: 416798), and The Nakuru County (Ref: NCG/CDMS/GEN.VOL1/299).

Additionally, written consent was sought from farm workers. No coercion of any kind was exerted to any of the participants, while participants asking to pull out from the study would be let totally free.

All the farm workers present during that particular working day shift were briefed about the study. An information sheet was given to each farm worker for more information; this sheet contained all relevant information deemed relevant for the prospective farmers to know regarding this study.

Thirty-three (33) trained research assistants explained the information on an individual level to farm workers who met the inclusion criteria and helped them to understand, complete and sign the consent form. Research assistants took each participant through the Nordic Musculoskeletal Questionnaire (NMQ). The research assistants filled the NMQ.

Relationship between WRMSDs risk and socio-demographic characteristics: There was no relationship between WRMSDs risk and socio-demographic characteris- Socio-demographic tics as shown in Table 2.

Table 2: Relationship between work-related risk and socio$(n=184)$. demographic characteristics

Those who will filled the NMQ to indicate that there was a presence of musculoskeletal disorders on any area of the body in the last 12 months proceeded to be assisted to fill the Rapid Entire Body Assessment. Research assistants had been contracted due to the initial perception of low educational levels among farm workers.

\section{RESULTS}

Prevalence of musculoskeletal disorders: The majority of the 270 respondents, namely 184 (68.1\%), reported having experienced discomfort in either one or more of their body areas over the previous one year while 86 (31.9\%) did not experience any WRMSDs.

The risk for development of musculoskeletal disorders: The Rapid Entire Body Assessment method was used to determine the level of risk of WRMSDs. Respondents were classified as having 'Medium rish', 'High risk', and 'Very High risk'. Most of the respondents ( $n=80,43.5 \%$ ) were in the 'High' risk category, as shown in Table 1.

Table 1: Work-related risk score of respondents with musculoskeletal disorders ( $n=184)$.

\begin{tabular}{ccc}
\hline & \multicolumn{2}{c}{ WRMSDs } \\
\cline { 2 - 3 } Risk Score & Frequency & Percent \\
\hline Medium Risk & 49 & 26.6 \\
High Risk & 80 & 43.5 \\
Very High Risk & 55 & 29.9 \\
\hline Total & $\mathbf{1 8 4}$ & $\mathbf{1 0 0}$ \\
\hline
\end{tabular}

\begin{tabular}{|c|c|c|c|c|c|}
\hline \multirow{2}{*}{$\begin{array}{c}\text { Socio-demographic } \\
\text { Characteristics }\end{array}$} & \multicolumn{3}{|c|}{ Level of Risk } & \multirow[t]{2}{*}{ Total } & \multirow[t]{2}{*}{ p-Value } \\
\hline & Medium ( $n=49$ ) & High $(n=80)$ & Very High $(n=55)$ & & \\
\hline \multicolumn{6}{|l|}{ Gender } \\
\hline Male & 25 (29.1\%) & $32(37.2 \%)$ & $29(33.7 \%)$ & 86 & \multirow{2}{*}{0.271} \\
\hline Female & $24(24.5 \%)$ & $48(49.0 \%)$ & $26(26.5 \%)$ & 98 & \\
\hline \multicolumn{6}{|l|}{ Age (Years) } \\
\hline$<30$ & 7 (63.6\%) & $3(27.3 \%)$ & 1 (9.1\%) & 11 & \multirow{4}{*}{0.052} \\
\hline $30-39$ & $10(16.1 \%)$ & $32(51.6 \%)$ & $20(32.3 \%)$ & 62 & \\
\hline $40-49$ & $29(29.6 \%)$ & $40(40.8 \%)$ & $29(29.6 \%)$ & 98 & \\
\hline$\geq 50$ & 3 (23.0\%) & $5 \quad(38.5 \%)$ & 5 (38.5\%) & 13 & \\
\hline \multicolumn{6}{|l|}{ Weight (Kgs) } \\
\hline$\leq 60$ & $18(29.0 \%)$ & $27(43.6 \%)$ & $17(27.4 \%)$ & 62 & \multirow{4}{*}{0.833} \\
\hline $61-80$ & $29(25.4 \%)$ & 48 (42.1\%) & $37(32.5 \%)$ & 114 & \\
\hline $81-100$ & $2(28.6 \%)$ & $4 \quad(57.1 \%)$ & 1 (14.3\%) & 7 & \\
\hline$>100$ & $0(0 \%))$ & 1 (100\%) & $0 \quad(0 \%)$ & 1 & \\
\hline \multicolumn{6}{|l|}{ Job designation } \\
\hline General Worker & $45(25.3 \%)$ & 79 (44.4\%) & $54(30.3 \%)$ & 178 & \multirow{2}{*}{0.077} \\
\hline Sprayer & $4 \quad(66.7 \%)$ & 1 (16.7\%) & $1(16.7 \%)$ & 6 & \\
\hline \multicolumn{6}{|c|}{ Length of time in worked (Years) } \\
\hline$\leq 5$ & $8(25.8 \%)$ & $14(45.2 \%)$ & $9(29.0 \%)$ & 31 & \multirow{6}{*}{0.904} \\
\hline $6-10$ & $17(21.8 \%)$ & $35(44.9 \%)$ & $26(33.3 \%)$ & 78 & \\
\hline $11-15$ & $17(32.1 \%)$ & $23(43.4 \%)$ & $13(24.5 \%)$ & 53 & \\
\hline $16-20$ & $5(35.7 \%)$ & $4(28.6 \%)$ & $5 \quad(35.7 \%)$ & 14 & \\
\hline $21-25$ & $1(16.7 \%)$ & $3(50.0 \%)$ & $2(33.3 \%)$ & 6 & \\
\hline$\geq 26$ & $1(50.0 \%)$ & $1(50.0 \%)$ & $0(0 \%)$ & 2 & \\
\hline
\end{tabular}


J. M. Munala, B. Olivier. Work-related risk associated with musculoskeletal disorders amongst flower farm workers in Kenya.

\section{DISCUSSION}

The Rapid Entire Body Assessment has a scoring classification: 'negligible risk', 'low risk', 'medium risk', 'high risk' and 'very high risk' scores. This present study reported 'medium risk' ( $n=49,26.6 \%)$, 'high risk' ( $n=80,43.5 \%)$, and 'very high risk' ( $n=55,29.9 \%)$ of sustaining WRMSDs. This present study reported presence of some risk across all respondents, as there were no 'negligible risk' and 'low risk' respondents as slotted on the REBA. By this results, they indicate that carrying out farm work predisposed respondents to WRMSD risk. This reported levels of risk is probably due to numerous reaching and twisting activities especially when picking ready flowers. The process of picking ready flowers involved repetitive motions of the wrist and hand joints over longer periods. Further, picking of ready flowers is a delicate task. It involves a lot of attention, which involves adoption and maintenance of awkward positions such as stooping and neck flexion. These tasks become more intensive during peak seasons since there is an increased demand of production. In addition, tasks such as transportation of the picked-flowers from the farm, transporting fertilizer to the farm, and even the routine farm nursery maintenance tasks exposed the farm workers to WRMSDs risk - this is also noted [1]. Lastly, the spraying activity involves loading the back with a knapsack sprayer containing pesticides or herbicides. On average, the knapsack is 18 to $20 \mathrm{~kg}$ in weight, this loading activity coupled with working for longer durations in a repetitive manner while twisting and reaching to all the areas of the farm predisposed the farm workers to WRMSD risk. The situation is worse when the flowers are still short and when they are in the nursery because, besides the loaded back with a knapsack sprayer and far-reaching maneuvers, the sprayers have to adopt a stooping position.

A systematic review [15] concurred to this present study that WRMSDs had a causal relationship with scoring a high work-related risk level if the activities involved biomechanical risk factors such as excessive repetition, heavy lifting and adopting awkward postures just as the respondents in this study. Similarly, a study among Chinese Obstetricians and Gynecologists showed that though there was a difference in risk level across workers, every worker had an absolute risk of developing WRMSDs due to their various working environments, psychosocial, individual, and postural factors [16] and this explains why farm workers from the two designations in this present study reported varying levels of WRMSD risk levels. A study on nurses' handling and transferring patients, reported that the level of workrelated risk increased when the nurses manually moved or lifted a patient - mostly if the patient were overweight or obese [34]. In a study [35] which included participants in eight work-station jobs, six institutions and three different industries reported various work-related risk levels across all eight work-stations.

Though a WRMSD study among dairy farmers in Iran did not report risk in percentages as this study did, they reported being unacceptably high risk since it was stratified as both 'high risk' and 'very high risk' [33]. This WRMSD risk stratification was almost similar to that of this study since in both populations, a lot of manual work was involved, assuming poor postural positions and carrying out of repetitive movements.

A study [28] among auto-mechanics had utilized the NMQ and the REBA method similar to this present study, reported that the workers were stratified as 'low', 'medium', 'high risk', and 'very high risk' at 5\%, 13.2\%, 24.2\%, $31.3 \%$, respectively. This was unlike in our study, because they were no 'low risk' workers reported in this present study. This was probably because the auto-mechanic industry job-needs were different from those of farm work and were less demanding, less repetitive in nature and did not entail adoption of poor postures especially for a long duration. The alluded probable reasons of differences in specific-industry demands might have led to three risk stratification in this present study and not two strata from a study [36] among hairdressers only reported 'high risk' (46\%) and 'very high risk' (14.9\%). An ergonomic study on the assessment of WRMSD risk among agriculture workers of Uttarakhand- 
J. M. Munala, B. Olivier. Work-related risk associated with musculoskeletal disorders amongst flower farm workers in Kenya.

India [32] precisely classified all respondents to be at high risk.

Even though all the five socio-demographic characteristics studied in this present study did not have a relationship with WRMSD risk. This could have been due the fact that REBA method only assessed physical factors such as forceful exertions and sustained posture, and not other factors such as psychosocial factors such as work-rest cycles, time pressure, and overall social support, and individual factors such as education levels which are considered as predisposing factors to WRMSD.

More general workers were classified as high and very high risk compared to the sprayers. This is attributed to the daily lifting and transporting of fertilizers, seedlings and the already-picked flowers tasks that they carry-out while on duty. They also stoop for prolonged durations while carrying out nursery and greenhouse activities. This clearly shows that, various job demands at workstations predispose workers to different levels of WRMSDs risk. These high levels of risk scores may also have been due to the little or no information on proper ergonomic guidelines, poor or no utilization of semi-mechanized tools, the need to work and produce in large volumes and so, increased working pace. The variation in levels of risk among designations and even industries and workstations was also noted in a studies [17-18, 35].

A few farm workers over 50 years of age $(n=5$, $38.5 \%)$ reported 'very high risk'. This is because, the perennial heavy lifting, prolonged bending posture, and repetitive movements done over a longer period worked negatively on the neural, skeletal and muscular system thus, decreased and or impaired function and or mobility. Even though this was a small number to draw conclusions from, the findings from this present study were also supported [4] which in their case. The age group aged between 46 to 55 years was the most affected. Although one study [11] used different age grouping from this present study, they found out the age group of 40-55 years had the most significant WRMSD risk. Their results were thereby almost similar to the results of this study. There was a correlation between an older age and a higher WRMSDs risk level [20,37]. Participants $(n=8)$ who weighed more than 80 kg reported a 'high risk' in developing WRMSDs than any other age cohorts. A higher weight among other reasons may cause a low self-esteem arising from psychosocial factors. Again, this is a small number to draw conclusions from. However, the results from the present study were similar with to Alghadir et al [18] on WRMSDs among dental professionals in Saudi Arabia who reported that weight was one of the socio-demographic characteristics that increased the risk of developing WRMSDs. Similarly, [17] a study on WRMSDs by women in a social rehabilitation center reported that subjects who required sick-leave showed a higher mean age, duration of working and weight.

Although our study did not find a link between the duration one has worked with work-related musculoskeletal risk, other studies such an occupational studies [19] and [20] reported that, among many other factors, the longer individuals were exposed to an occupation, the more likely they were exposed to WRMSD risk.

Limitations of the study: This study captured very few of the sprayers $(n=15)$ as opposed to the general workers $(n=255)$. By itself, the sprayers might therefore not have been represented well for the purposes of the subgroup analysis.

This study was only limited to data collection by the REBA as a sole method of assessing work-related musculoskeletal disorder risk. REBA method only assessed physical factors and not psychosocial and individual factors. Further studies should, therefore consider the use of more comprehensive tools that can capture these two factors.

Lastly, in this study only respondents with WRMSDs, as reported pain on NMQ proceeded to fill the REBAQ. Therefore, the construct validity of the REBAQ could not be determined and this aim was outside the scope of this study. Some respondents who did not report pain within the previous 12 months might have a high risk level of contracting WRMSDs as well.

\section{CONCLUSION}

This study was conducted to determine 
work-related risk associated with musculoskeletal disorders among flower farm workers flower farm workers involved in strenuous activities, repetitive movements, and awkward posture and overall farm maintenances are predisposed to work-related risk. However, no relationship between work-related risk of musculoskeletal disorders and socio-demographic factors could be found.

We recommend that further research is carried out to reduce the WRMSD risk by exploring causative relationships.

\section{ACKNOWLEDGEMENTS}

Special thanks to the Research Assistants (Physiotherapy students at The Kenya Medical Training College, Nakuru Campus) for the assistance they offered in data collection.

Special thanks also go to the overall administration of the Farm for the support they accorded this researcher during the data collection phase of this research.

Funding: This research received no specific grant from any funding agency in the public, commercial, or not-for-profit sectors.

Data Availability Statement: Data sharing is not applicable to this article as no new data were created or analysed in this study.

Disclaimer: The views and opinions expressed in this article are those of the authors and do not necessarily reflect the official policy or position of any affiliated agency of the authors.

\section{Conflicts of interest: None}

\section{REFERENCES}

[1]. Benos L, Tsaopoulos D, Bochtis D. A review on ergonomics in agriculture. Part I: Manual operations. Applied Sciences (Switzerland). 2020.

[2]. Nunes IL, Bush PM. Work-Related Musculoskeletal Disorders Assessment and Prevention. Ergon Syst Approach. 2011;1-31.

[3]. Vedovato TG, Monteiro I. Health Conditions and Factors Related to the Work Ability of Teachers. Ind Health. 2014;52(2):121-8.

[4]. Riccò M, Cattani S, Gualerzi G, Signorelli C. Work with visual display units and musculoskeletal disorders: A cross-sectional study. Med Pr. 2016;67(6):707-19.

[5]. Ganiyu S, Muhammad I, Olabode J, Stanley M. Patterns of occurrence of work-related musculoskeletal disorders and its correlation with ergonomic hazards among health care professionals. Niger J Exp Clin Biosci [Internet]. 2015;3(1):18. Available from: http://www.njecbonline.org/text.asp?2015/ 3/1/18/158153

[6]. Azim F. The prevalence of musculoskeletal disorders among madrassa teachers and students-a cross sectional study. Int J Physiother. 2016;3(31):100-5.

[7]. Shafti A, Lazpita BU, Elhage O, Wurdemann HA, Althoefer K. Analysis of comfort and ergonomics for clinical work environments. In: Proceedings of the Annual International Conference of the IEEE Engineering in Medicine and Biology Society, EMBS. 2016;1894-7.

[8]. Fathallah FA, Miller BJ, Miles JA. Low back disorders in agriculture and the role of stooped work: Scope, potential interventions, and research needs. Journal of Agricultural Safety and Health. 2008.

[9]. Darwish MA, Al-Zuhair SZ. Musculoskeletal pain disorders among secondary school Saudi female teachers. Pain Res Treat. 2013;2013.

[10]. National statistics. Work-related Musculoskeletal Disorder Statistics, Great Britain 2016. Heal Saf Exec. 2016,

[11]. Zoer I, Frings-Dresen MHW, Sluiter JK. Are musculoskeletal complaints, related work impairment and desirable adjustments in work age-specific? Int Arch Occup Environ Health. 2014;87(6):647-54.

[12]. Buckle P, Devereux J. Work-related neck and upper limb musculoskeletal disorders. Appl Ergon [Internet]. 2002;33:207-17. Available from: htt p://scholar.google.com/scholar? cluster $=5878916485553067887 \& \mathrm{hl}=\mathrm{en} \# 0$

[13]. Nunes IL. FAST ERGO-X - A tool for ergonomic auditing and work-related musculoskeletal disorders prevention. Work. 2009;34(2):133-48.

[14]. Ekpenyong CE, Inyang UC. Associations between worker characteristics, workplace factors, and work-related musculoskeletal disorders: A crosssectional study of male construction workers in Nigeria. Int J Occup Saf Ergon. 2014;20(3):447-62.

[15]. Costa BR, Vieira ER. Risk factors for work-related musculoskeletal disorders: A systematic review of recent longitudinal studies. Vol. 53, American Journal of Industrial Medicine. 2010. p. 285-323.

[16]. Wang J, Cui Y, He L, Xu X, Yuan Z, Jin X, et al. Workrelated musculoskeletal disorders and risk factors among Chinese medical staff of obstetrics and gynecology. Int J Environ Res Public Health. 2017;14(6):1-13.

[17]. Pastre EC, Carvalho Filho G, Pastre CM, Padovani CR, Almeida JS De, Netto Jr J. Work-related musculoskeletal complaints by women in a social rehabilitation center. Cad saude publica / Minist da Saude, Fund Oswaldo Cruz, Esc Nac Saude Publica. 2007;23(11):2605-12.

[18]. Alghadir A, Zafar H, Iqbal ZA. Work-related musculoskeletal disorders among dental professionals in Saudi Arabia. J Phys Ther Sci [Internet]. 2015;27(4):1107-12. Available from: http:// w w w. pu b m ed central.nih.gov/ articlerender.fcgi?artid $=4433988 \&$ tool $=$ pmcentrez \&rendertype=abstract 
J. M. Munala, B. Olivier. Work-related risk associated with musculoskeletal disorders amongst flower farm workers in Kenya.

[19]. Tinubu BMS, Mbada CE, Oyeyemi AL, Fabunmi AA. Work-related musculoskeletal disorders among nurses in Ibadan, South-west Nigeria: a crosssectional survey. BMC Musculoskelet Disord [Internet]. 2010;11(1):12. Available from: http:// www.pubmedcentral.nih.gov/articlerender.fcgi? artid $=2823665 \&$ tool $=$ pm centrez \& rendertype =abstract $\% 255 \mathrm{Cnhttp}: / /$ bmcmusculoskeletdisord. biomedcentral.com/articles/10.1186/1471-247411-12

[20]. Shuai J, Yue P, Li L, Liu F, Wang S. Assessing the effects of an educational program for the prevention of work-related musculoskeletal disorders among school teachers. BMC Public Health. 2014;

[21]. Tebyetekerwa M, Akankwasa NT, Marriam I. The Current Working Conditions in Ugandan Apparel Assembly Plants. Saf Health Work. 2017;8(4): 378-85.

[22]. Fathallah FA. Musculoskeletal disorders in labor-intensive agriculture. Appl Ergon. 2010;41(6):738-43.

[23]. Abdalla S, Apramian SS, Cantley LF, Cullen MR. Occupation and risk for injuries.

[24]. Osborne A, Blake C, Meredith D, Kinsella A, Phelan J, Mcnamara J, et al. Work-related musculoskeletal disorders among Irish farm operators. Am J Ind Med. 2013;56(2):235-42.

[25]. Cochran WG. Sampling techniques. Vol. 20, Wiley publisher. 1977. 448 pages.

[26].Crawford JO. The Nordic Musculoskeletal Questionnaire. Occup Med (Chic III) [Internet]. 2007;57(4):300-1. Available from: https:// academic.oup.com/occmed/article-lookup/doi/ 10.1093/occmed/kqm036

[27]. Middlesworth M. A Step-by-Step Guide to the REBA Assessment Tool. Dipetik Mei. 2015;17.

[28]. Moradi M, Poursadeghiyan M, Khammar A, Hami $M$, Darsnj A, Yarmohammadi H. REBA method for the ergonomic risk assessment of auto mechanics postural stress caused by working conditions in Kermanshah (Iran). Ann Trop Med Public Heal. 2017.

[29]. Hignett S, McAtamney L. Rapid Entire Body Assessment (REBA). Vol. 31, Applied Ergonomics. 2000. p. 201-5.
[30]. Kaewdok T, Sirisawasd S, Taptagaporn S. Agricultural risk factors related musculoskeletal disorders among older farmers in Pathum Thani Province, Thailand. Journal of agromedicine. $2020 \mathrm{Jul}$ 18:1-8.

[31]. Das B, Ghosh T, Gangopadhyay S. Assessment of ergonomic and occupational health-related problems among female prawn seed collectors of sunderbans, West Bengal, India. Int J Occup Saf Ergon. 2012.

[32]. Ojha P, Kwatra S. An ergonomic study on the assessment of work related musculoskeletal disorder risks among agriculture workers of Uttarakhand, India. Int J Sci Eng Res. 2014;5(1): 188-91.

[33]. Taghavi SM, Mokarami H, Ahmadi O, Stallones L, Abbaspour A, Marioryad H. Risk factors for developing work-related musculoskeletal disorders during dairy farming. Int J Occup Environ Med. 2017

[34]. Choi SD, Brings K. Work-related musculoskeletal risks associated with nurses and nursing assistants handling overweight and obese patients: A literature review. Work [Internet]. 2016;53:439-48. Available from: http://www.ncbi.nlm.nih.gov/ pubmed/26835850

[35]. Oakman J, Chan S. Risk management: Where should we target strategies to reduce work-related musculoskeletal disorders? Saf Sci. 2015;73:99105.

[36]. Mahdavi S, Mahdavi M, Safary M, Rashidi R, Dehghani T, Kosari M. Evaluation of the risk of musculoskeletal disorders using Rapid Entire Body Assessment among hairdressers in Khorramabad, Iran, in 2014. J Occup Heal Epidemiol. 2013.

[37]. Okunribido OO, Wynn T, Lewis D. Are older workers at greater risk of musculoskeletal disorders in the workplace than young workers? - A literature review. Occupational Ergonomics. 2011.

$$
\begin{aligned}
& \text { How to cite this article: } \\
& \text { Munala J. M, Olivier B. Work-related risk associated with musculoskeletal } \\
& \text { disorders amongst flower farm workers in Kenya. Int J Physiother Res } \\
& \text { 2021;9(3):3817-3824. DOI: } 10.16965 / \text { ijpr.2021.120 }
\end{aligned}
$$

